\title{
Filigrane
}

Écoutes psychothérapiques

\section{La pensée qui soigne. Que savons-nous du pouvoir des émotions? 1, de Monique Brillon}

\section{André Renaud}

Volume 15, numéro 2, automne 2006

URI : https://id.erudit.org/iderudit/014468ar

DOI : https://doi.org/10.7202/014468ar

Aller au sommaire du numéro

Éditeur(s)

Revue Santé mentale au Québec

ISSN

1192-1412 (imprimé)

1911-4656 (numérique)

Découvrir la revue

Citer ce compte rendu

Renaud, A. (2006). Compte rendu de [La pensée qui soigne. Que savons-nous du pouvoir des émotions ? 1, de Monique Brillon]. Filigrane, 15(2), 94-95.

https://doi.org/10.7202/014468ar d'utilisation que vous pouvez consulter en ligne. 


\section{La pensée qui soigne. Que savons-nous du pouvoir des émotions ? ${ }^{1}$, de Monique Brillon}

\section{andré renaud}

$\mathrm{D}$ ans un premier essai, publié en 1998 et intitulé Ces pères qui ne savent pas aimer et les femmes qui en souffrent, Monique Brillon expliquait, en s'appuyant sur la psychanalyse, le rôle du père dans le développement de l'identité de la femme. Sa thèse était illustrée par de nombreux cas cliniques.

L'essai qui vient de paraître porte sur la nature psychosomatique de l'être humain. Depuis plusieurs années, Monique Brillon, docteure en psychologie et psychothérapeute psychanalytique, s'intéresse aux problèmes de santé et à leurs liens avec les conflits psychiques, liens plus souvent inconscients que conscients. Dre Brillon présente de façon posée et claire comment le corps entretient des relations assez nettes et efficaces, même si elles sont très complexes, avec les émotions et la pensée, et comment la psyché peut influencer en retour le fonctionnement du corps.

Non, il ne s'agit pas d'un livre sur la pensée positive, ni d'une quelconque méthode de guérison. L'auteure démontre comment l'expérience de vie s'enregistre dans le corps et comment le travail de l'appareil psychique est de traduire en matériel psychique les influx nerveux, les sécrétions, les contractions, et autres phénomènes physiologiques éprouvés dans et par le corps. Une émotion est d'abord une réaction - physiologique, endocrinologique, neurologique ou autre - et celle-ci doit être traduite en significations psychiques pour donner un sens à l'expérience vécue. Cette signification, plus souvent inconsciente, parfois préconsciente et, plus rarement encore, consciente, pourra en retour favoriser un meilleur fonctionnement organique, un corps en meilleure santé.

La mémoire, les émotions font le pont entre les fonctions organiques, la pensée et le travail d'élaboration psychique d'où découle la signification personnelle de l'expérience de vie. Cependant, la circulation des émotions, des expériences mémorisées peut être parfois perturbée, tronquée, paralysée et le vécu corporel ne parvient pas alors, ou mal ou trop partiellement, à une traduction psychique. C'est alors que sont créés des embâcles, des stagnations qui soumettent le corps et ses différentes fonctions à des tensions, des stress et des malaises qui deviennent des prédispositions aux maladies.

Il n'y a pas à proprement parler de maladies psychosomatiques. C'est l'être humain qui est psychosomatique. Le corps fonctionne comme un tout et tout ce qui pénètre le psychique est d'abord passé par le corps. La question est de savoir quelle proportion de ce qui s'inscrit dans le corps parvient à être traduite en 
significations psychiques et quelle est la qualité de cette seconde inscription. Le bon fonctionnement du corps relève du bon fonctionnement de la psyché autant que de l'alimentation, de l'exercice, d'un environnement sain, etc.

Encore une fois, l'auteure illustre ses propos par des vignettes cliniques appropriées et des exemples bien choisis de la vie quotidienne. Bien que le livre s'adresse à un public large, il apparaît évident que les médecins, les psychologues et autres intervenants en santé y puiseront non seulement matière à réflexion, mais y découvriront des dimensions et des applications utiles. Les spécialistes seront interpellés dans leurs pratiques quotidiennes, routinières qui peuvent avoir tendance à s'automatiser, se stéréotyper. Voilà une lecture qui rappelle que l'être humain n'est pas qu'un corps, qu'une belle machine, mais qu'il est aussi un être sensible, voire souvent susceptible et encore plus souvent à son insu. Le dernier essai de Monique Brillon nous le rappelle et nous y sensibilise d'une façon intelligente et signifiante.

andré renaud 1180 , charles albanel sainte foy, qc g1x $4 \mathrm{t} 9$

renauda@videotron.ca

\section{Note}

1. Monique Brillon, Ph. D., 2006, La pensée qui soigne. Que savons-nous du pouvoir des émotions?, Montréal, Les Éditions de l'homme, 2006, 255 p. 